\title{
Extremum seeking x-ray position feedback using power line harmonic leakage as the perturbation
}

\author{
S. Zohar, D. J. Kissick, N. Venugopalan, C. M. Ogata, O. Makarov, \\ S. Stepanov, and R. F. Fischetti \\ Advanced Photon Source, Argonne National Laboratory, 9700 South Cass Avenue, \\ Building 436, Lemont, Illinois 60439, USA \\ (Received 30 May 2016; published 12 September 2016)
}

\begin{abstract}
Small x-ray beam sizes necessary for probing nanoscale phenomena require exquisite stability to prevent data corruption by noise. One source of instability at synchrotron radiation x-ray beamlines is the slow detuning of x-ray optics to marginal alignment where the onset of clipping increases the beam's susceptibility to higher frequency position oscillations. In this article, we show that a $1 \mu \mathrm{m}$ amplitude horizontal x-ray beam oscillation driven by power line harmonic leakage into the electron storage ring can be used as perturbation for horizontal position extremum seeking feedback. Feedback performance is characterized by convergence to $1.5 \%$ away from maximum intensity at optimal alignment.
\end{abstract}

DOI: 10.1103/PhysRevAccelBeams.19.092801

\section{INTRODUCTION}

Synchrotron radiation is a powerful tool that has been used to explore the structural [1-3], electronic [4-6], and chemical [7] properties of both organic and inorganic materials. In recent years, the promise of nanoscience for new exciting technologies has motivated the development of $\mathrm{x}$-ray optics that can better resolve nanoscale phenomena [8-10]. While x-ray optics and synchrotron instrumentation have seen tremendous progress, beam stabilization methods have received less attention. As X-ray beam sizes are reduced to resolve increasingly smaller regions, the tolerance for beam instabilities will be increasingly constrained.

Noise present in experiments decreases data acquisition rates. From amongst the various sources of noise present at $\mathrm{x}$-ray beam lines, beam position and intensity instabilities are particularly unfavorable. Compared to electronic interference and photo-detector dark current, positional instabilities are encoded onto the beam. This mitigates the benefit of electronic noise suppression techniques such as photon counting and lock-in background subtraction. To alleviate this problem, significant effort has been invested in studying both feedforward suppression and feedback correction of beam instabilities originating in both $\mathrm{x}$-ray optics [11-18] and the particle beam in the storage ring [19-21]. In previous studies [11-18], double crystal monochromator (DCM) intensity stabilization was achieved using extremum seeking feedback (ESF) [22] to align the DCM 2nd crystal angle to the Bragg peak maximum. While this approach maximizes the intensity immediately

Published by the American Physical Society under the terms of the Creative Commons Attribution 3.0 License. Further distribution of this work must maintain attribution to the author(s) and the published article's title, journal citation, and DOI. downstream from the DCM, the beam intensity at the sample may not be optimal. Recent experiments have demonstrated that oscillations introduced onto the DCM 2nd crystal angle required for ESF intensity optimization of the DCM intensity could simultaneously be used to stabilize the vertical beam position by using ESF optimization of intensity through an aperture immediately upstream from the sample [23]. This approach is favorable compared to position feedback based on quadrant diode beam position monitors (BPM) due to reduced sensitivity to both detector dark currents and parallax errors arising from thermally induced mechanical drifts between the BPM and the source. In the conclusion, it was predicted that the intensity gradient required for X-ray beam position ESF control could be estimated by using $\mathrm{X}$-ray beam oscillations originating in the storage ring.

In this article, we report the implementation of ESF for controlling the $\mathrm{x}$-ray beam horizontal position using a $1.8 \mathrm{kHz}$ oscillation arising from power line harmonic leakage into the electron storage ring as the perturbation. The ability to implement ESF control without introducing additional perturbations is attractive because no disturbance is added to the beam. Efforts at applying ESF without introduced perturbations is the primary thrust of stochastic extremum seeking feedback [24,25], and has found application in optimizing dc-dc converter switching for photovoltaics [26] and hydrogen fuel consumption in polymer electrolyte membrane fuel cells [27]. In this work, horizontal X-ray beam position oscillations originating in the electron beam are measured downstream from the DCM. The horizontal position oscillation phase is used as the reference for determining the intensity spatial gradient at the sample. The estimate of this gradient enables ESF control for maximizing the beam intensity through an aperture. Feedback performance here is characterized by 
convergence to within 7\% of the peak position normalized to FWHM and $1.5 \%$ of max intensity respectively.

\section{EXPERIMENT}

Experiments were conducted at the Advanced Photon Source (APS) at GM/CA-XSD, beamlines 23-ID-D and 23-ID-B. The experimental layout is shown in Fig. 1. $\mathrm{X}$-rays are produced at this beamline by accelerating the electron beam in the storage ring using a magnetic field generated by an insertion device [28]. The DCM selects a narrow energy $\left(\Delta E / E=2 \times 10^{-4}\right)$ bandwidth from the undulator emission spectrum. The beam is focused using vertical and horizontal focusing mirrors (VFM, HFM) arranged in a Kirkpatrick-Baez geometry. Each mirror sits in a cradle supported by flex pivots. A piezo actuator pushes on the mirror assembly driving the mirror steering angle. Slip contact between the mirror cradle and the piezo actuator is maintained by a compression spring. A collimator immediately upstream from the sample reduces background scatter and improves spatial resolution by reducing the beam size to less than the sample size [29-31]. Beam intensity downstream from the sample is detected using a silicon PIN junction diode. Intensity at this detector is maximized when the beam is optimally aligned.

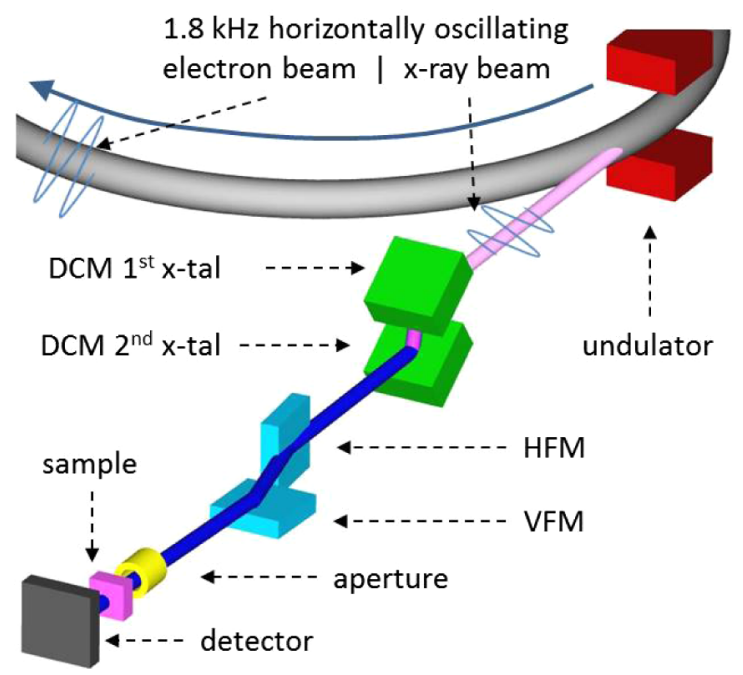

FIG. 1. Schematic of the electron storage ring, undulator source, and beamline optics. Radio frequency (rf) accelerator cavities (not shown) replenish energy lost to X-rays generated by the bending magnet or undulator sources. The $1.8 \mathrm{kHz}$ power line harmonic leaks through the high voltage rf power supplies driving a $1 \mu \mathrm{m}$ amplitude horizontal oscillation in the particle beam. A narrow energy band of $\mathrm{x}$-rays generated in the undulator are selected by the dual crystal monochromator (DCM). The horizontal focusing mirror (HFM) and vertical focusing mirror VFM steer and focus the beam at the sample. The collimating aperture reduces the beam size before passing through the sample. The intensity detector in this demonstration is a silicon PIN junction diode.
The controls diagram for the horizontal position ESF is shown in Figure 2. The beam position downstream from the DCM is measured using a quadrant diode BPM that detects $\mathrm{x}$-ray backscatter from a silver foil. The $1.8 \mathrm{kHz}$ horizontal beam oscillation is phase locked using an analog phase locked loop (PLL) whose voltage controlled oscillator (VCO) is centered at $1.8 \mathrm{kHz}$. The PLL output is used as an external reference for the lock-in amplifier (LIA). The lock-in amplifier detects the phase of the beam intensity oscillations at the sample with respect to the phase locked BPM signal. When the $\mathrm{x}$-ray beam horizontal position is not optimally aligned, intensity fluctuations detected downstream from the sample are either in or 180 degrees out of phase with respect to the $1.8 \mathrm{kHz}$ reference. The LIA output is then integrated using a field programmable gate array (FPGA) and fed into the HFM steering piezo controller.

\section{THEORY}

In this section, an analysis applying extremum seeking feedback to x-ray optics steering is adapted from Ref. [23] to include the HFM steering time response and beam size. The intensity of a Gaussian beam at the sample after passing through a collimating aperture can be approximated as

$$
I=I_{0}\left[-\frac{1}{2}\left(\frac{\psi-\psi_{0}-\psi_{n}(\omega)}{\sigma}\right)^{2}+1\right]
$$

where $\psi$ is the beam horizontal angle, $\psi_{0}$ is the beam horizontal angle at optimal alignment, $I_{0}$ is the optimal intensity, $\sigma$ is the convolution of the beam and aperture width, and $\psi_{n}(\omega)$ is random beam motion as a function of frequency $\omega$. The horizontal beam angle is

$$
\psi=\psi_{H}+\psi^{\prime} \sin \left(\omega_{r} t\right)
$$

where $\psi_{H}$ is the HFM steering angle and $\psi^{\prime}$ and $\omega_{r}$ are the horizontal beam oscillation amplitude and $1.8 \mathrm{kHz}$ frequency originating from electron beam motion respectively. The LIA detects the signal from the intensity monitor and multiplies it by the $1.8 \mathrm{kHz}$ reference. The LIA output is then integrated resulting in

$$
\dot{V}=\left[g I+V_{n}(\omega)\right] \sin \left(\omega_{r} t\right)
$$

where the right-hand side is the output from the LIA when the internal time constant is very small, $V$ is the piezo drive voltage output from the FPGA integration stage, $V_{n}(\omega)$ is random noise originating in the intensity sensor after accounting for all gain stages, and $g$ is the total gain contributed from lock-in amplifier, integrator, and sensor amplifier. The units of $g$ have an additional inverse seconds component due to the units of integrator gain.

Combining Eqs. (1), (2), and (3), removing terms oscillating at or faster than $\sin (\omega t)$, and neglecting effects of detector noise and random beam motion results in 


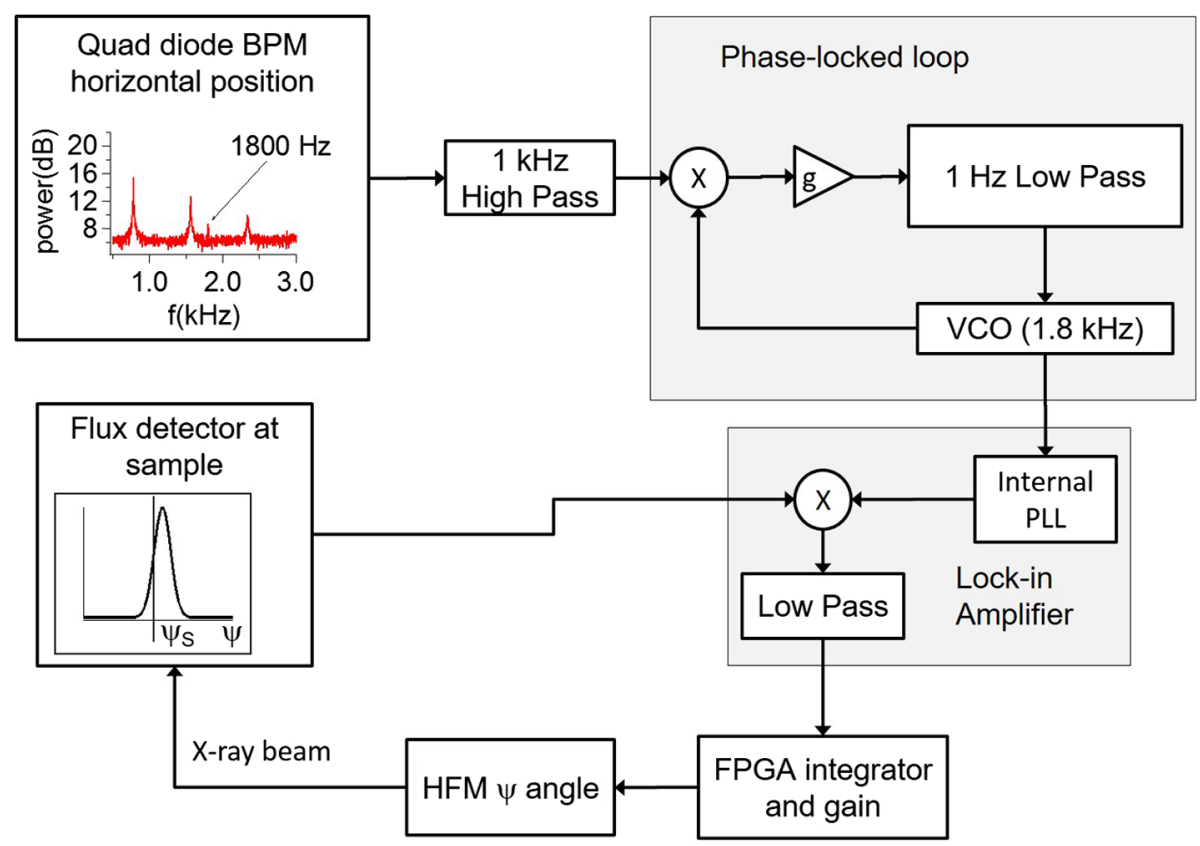

FIG. 2. The phase reference is extracted from the horizontal beam position monitor (BPM) downstream from the DCM using a phase locked loop (PLL) with the voltage controlled oscillator (VCO) centered at $1.8 \mathrm{kHz}$. The phase locked signal is then fed into the lock-in amplifier (LIA) external reference input. The LIA detects the phase between the reference and intensity oscillations using a digital mixer (multiplication sign). The resultant signal from the LIA is then digitally integrated using a field programmable gate array (FPGA). The integrated signal out of the FPGA is used to control the HFM piezo actuator, steering the beam towards optimal intensity.

$$
\dot{V}=\frac{-g I_{0} \psi^{\prime}}{\sigma^{2}}\left(\psi_{H}-\psi_{0}\right) .
$$

The removal of $\sin \left(\omega_{r} t\right)$ and its harmonics is the crux of the extremum seeking feedback derivation, and a rigorous justification using nonsingular perturbation theory can be found in Refs. [22,32]. Empirically, the HFM steering angle responds to the driving voltage $V$ according to

$$
\ddot{\psi}_{H}+\gamma \dot{\psi}_{H}+\omega_{0}^{2}\left(\psi_{H}-q V\right)=0
$$

where $\omega_{0}$ is the $\sim 30 \mathrm{~Hz}$ HFM-spring assembly resonant frequency, $\gamma$ is a phenomenological damping term, and $q$ is a constant that converts the applied control voltage into the target HFM steering angle. Combining Eqs. (4) and (5) yields

$$
\dddot{\psi}_{H}+\gamma \ddot{\psi}_{H}+\omega_{0}^{2}\left[\dot{\psi}_{H}+\frac{q g I_{0} \psi^{\prime}}{\sigma^{2}}\left(\psi_{H}-\psi_{0}\right)\right]=0 .
$$

This equation is a 3rd order linear differential equation with solutions of the form

$$
\psi_{H}=\psi_{0}+\sum_{n} C_{n} e^{\lambda_{n} t}
$$

where the convergence rate, $\lambda_{n}$, are the roots of the characteristic polynomial

$$
\lambda^{3}+\gamma \lambda^{2}+\omega_{0}^{2} \lambda+\frac{\omega_{0}^{2} q g I_{0} \psi^{\prime}}{\sigma^{2}}=0
$$

and $C_{n}$ are constant coefficients.
In the large spring stiffness limit, $\omega_{0} \gg \ddot{\psi}_{H}$, the convergence rate is $\lambda=q g I_{0} \psi^{\prime} / \sigma^{2}$. Varying the electron beam current, DCM wavelength, sample absorption, or any other parameter that increases (decreases) the intensity downstream from the sample also increases (decreases) the convergence rate. The effects of detector noise and random position motion are accounted for by assuming a solution of the form in Eq. (7) and treating $V_{n}$ and $\psi_{n}$ as driving terms. Doing so results in the steady state HFM steering angle of

$$
\psi_{H}(\omega)=\psi_{0}+\frac{\psi_{n}(\omega)}{1+i \omega / \lambda}+\frac{q V_{n}\left(\omega-\omega_{r}\right)}{\lambda+i\left(\omega-\omega_{r}\right)} .
$$

The effect of random beam motion and detector noise on intensity can be observed by reevaluating Eq. (1) using Eqs. (2) and (9). When the random beam position motion is slow $(\omega \ll \lambda)$, the feedback maintains alignment. As the random beam motion frequency increases, feedback's ability to maintain alignment rolls off, and the intensity measured through the aperture fluctuates. The effect of electronic noise is more involved. When the detector noise is close to $1.8 \mathrm{kHz}$, the feedback circuits responds as if the HFM is misaligned and drives HFM steering out of alignment. This effect is more pronounced as the electronic gain is increased.

As the spring stiffness is reduced, the HFM's moment of inertia, natural resonance, and damping contribute to the dynamics. In this regime the convergence rate may be 
imaginary or have positive values and the Hurwitz stability criterion [22] is no longer guaranteed. Intuitively, the HFM response to compensation of steady state oscillations above the HFM resonant frequency is $\pi$ out of phase with the compensating drive. In such a situation, feedback's attempts to counter motion above the HFM resonance frequency is counterproductive and drives the HFM steering further away from alignment. When the feedback gain is sufficiently high, the oscillation amplitude above the HFM resonance frequency grows. The analysis presented only accounts for the mirror resonance and feedback electronics. A more thorough treatment taking the bandwidth response of each element into account would be required for a more complete picture.

\section{RESULTS}

Impulse response curves from applied HFM steering deflections away from optimal alignment are shown in Fig. 3. Convergence rates are observed to increase with the feedback gain factor. The initially flat response to applied step deflections indicate the convergence curves are not purely exponential and that the large spring stiffness limit $\omega_{0} \gg \ddot{\psi}_{H}$ is not satisfied. Instead, the relaxation rate takes complex values and the convergence curves are more
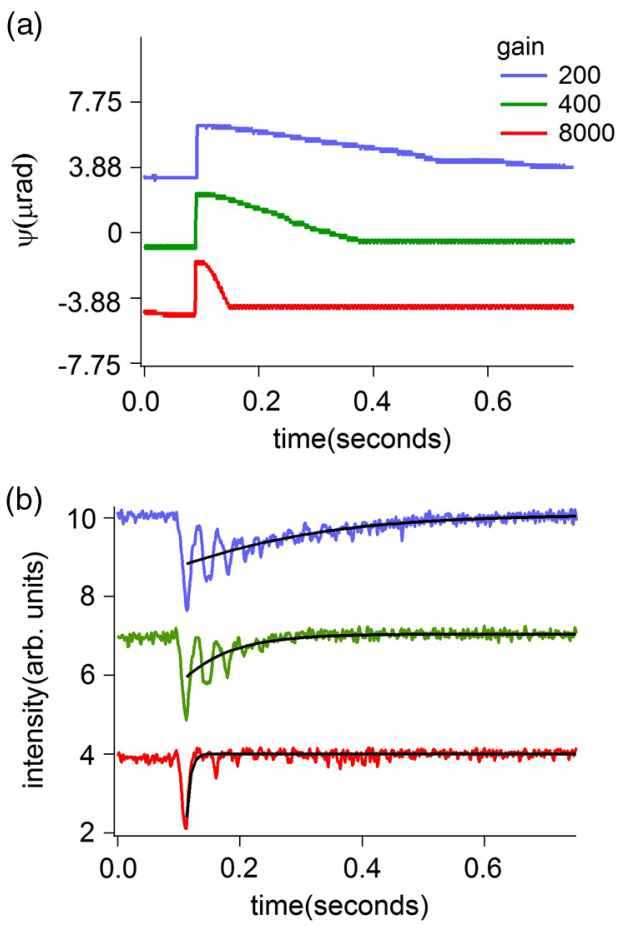

FIG. 3. (a) Unencoded HFM piezo steering angle set value and (b) sample intensity convergence times as a function of feedback gain. Curves have been offset for clarity. The convergence time decreases with increasing feedback gain. The $30 \mathrm{~Hz}$ oscillations in the diode intensity are due to the HFM mechanical resonance at $30 \mathrm{~Hz}$ that is excited by applying a step deflection to the HFM steering angle set value. The black curves are best fits to an exponential.
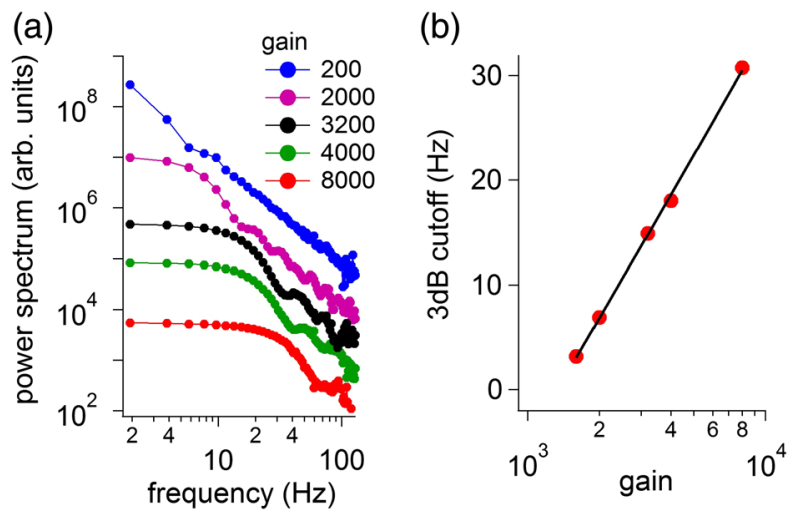

FIG. 4. (a) The power spectrum of the HFM steering angle set value to step deflection is shown in Fig. 3 for varying feedback gain. Curves have been offset for clarity. (b) The $3 \mathrm{~dB}$ cutoff increases linearly with feedback electronic gain.

accurately described by the sum of an exponential with exponentially decaying sinusoids. To characterize this convergence rate, we show the $3 \mathrm{~dB}$ cutoff of the response power spectrum as a function of feedback gain in Fig. 4(a). The $3 \mathrm{~dB}$ cutoff exhibits a linear dependence on the feedback gain [Fig. 4(b)]. The $\sim 30 \mathrm{~Hz}$ oscillations that appear in the intensity impulse response are due to the HFM mirror mechanical resonance. Feedback gain corresponding to the $3 \mathrm{~dB}$ cutoff above this resonant frequency are not stable and sustained oscillations occur. While the $\mathrm{X}$-ray intensity converges back to the initial value, the $\mathrm{X}$-ray beam steering does not converge back to initial angle set value due to piezo hysteresis. Feedback converges to within $7 \%$ away from peak position normalized to horizontal beam size FWHM and $1.5 \%$ away from peak intensity.

The x-ray beam horizontal oscillation amplitude at both $360 \mathrm{~Hz}$ and $1800 \mathrm{~Hz}$ in the vicinity of the sample is $95 \mathrm{~nm}$, and was calculated by comparing the lock-in signal to the transmission signal. Further details for this procedure can be found in the appendix. Accounting for HFM focal demagnification yields an oscillation amplitude of $\sim 1$ um at the source. This is less than $1 \%$ of the $650 \mu \mathrm{m}$ FWHM beam source size at the insertion device.

\section{DISCUSSION}

There are a number of benefits and limitations to this feedback approach. The foremost benefit is the increased convergence rate and signal to noise ratio (SNR) with decreasing beam size. This can be observed by the beam width that appears in the denominator of Eq. (8). This indicates that the convergence time for tightly focused beams have faster convergence rates than larger beams with the same area integrated flux. This will be especially beneficial for upcoming 4th generation sources that will have lower emittance and smaller beams. Another potential benefit of this feedback method is the ability to steer the electron beam upstream the insertion device. Such an 
approach can determine the intensity / position gradient by correlating white beam intensity fluctuations measured using grazing incidence fluorescence detectors with the electron beam position in the storage ring.

The foremost limits are the perturbation frequency and amplitude. At its core, ESF is a gradient based optimization technique, with the gradients estimated on millisecond times scales by relying on a naturally occurring or applied perturbations to explore the designated parameter space. With phase locking electronics, several oscillations are required to determine the phase and amplitude, and more oscillations are required for improved SNR. In this experiment, the perturbation frequency limits the gradient determination rate to a lower bound of $1 / 1800 \mathrm{~Hz}$, or $\sim 0.6 \mathrm{~ms}$. Sensor bandwidth due to capacitive coupling across the silicon depletion region limits the maximum perturbation frequency that can be used; this can range from as low as several $\mathrm{kHz}$ for large area silicon PN junction diodes, to up to several GHz for small area high speed silicon PIN junction diodes. The source oscillation amplitude also limits the convergence rate and SNR. Whereas standard extremum seeking feedback can increase both SNR and convergence rates by increasing the perturbation oscillation amplitude, using naturally occurring oscillations puts the experiment at the mercy of the system; when the natural oscillation amplitude is very small, the feedback converges very slowly.

Another limitation is the gain setting and electronic noise. The feedback convergence rate increases with increasing gain. As the feedback gain is increased to larger values, instabilities due to small negative and positive real components appearing in the roots of Eq. (8) allow for noise driven sustained ringing and spurious oscillation, respectively. Increasing the gain also increases the feedback noise due to amplification of electronic noise. For reference, it is observed that a gain of 3000x split between 100x in the lock-in amplifier and 30x in the integrator stage excites a position noise of $180 \mathrm{nrad} \mathrm{FWHM}$, corresponding to a virtual position noise of $0.6 \mu \mathrm{m}$ at the source. One way to alleviate this is by balancing the gain between the FPGA integration and lock-in amplifier to exploit the 12-bit resolution of the FPGA ADC and provide a dead band that suppresses unwanted driving at the expense of reduced accuracy.

Finally, we note that power line transients destabilize the external analog PLL from its lock on the $1.8 \mathrm{kHz}$, intermittently driving the beam out of alignment. More sophisticated FPGA electronics that suppress feedback when the phase lock is lost will be required to prevent transient driven instabilities that occur intermittently on time scales of tens of minutes.

In this experiment, a naturally occurring phase stable $1.8 \mathrm{kHz}$ oscillation present in the electron storage ring has been used to determine the intensity position gradient. ESF control was also successfully implemented using a $360 \mathrm{~Hz}$ power line harmonic as the reference. Full implementation of stochastic ESF, however, does not require the perturbation to be phase stable. As electron beam oscillations in the storage ring continue to be reduced, phase stable oscillations may have insufficient amplitude for driving ESF. At that point, ESF stabilization will need to adopt a full stochastic approach using random beam motion as opposed to phase stable oscillations. Such an approach would improve convergence rates and SNR by averaging intensity-position gradients simultaneously determined from multiple beam motion frequencies instead of from a single phase stable frequency.

Together with previous work [23], this work constitutes a feedback control in both transverse directions. Intensity fluctuations frequencies are identified with their respective position oscillation axes using time resolved sample and DCM intensities gathered prior to this experiment.

\section{CONCLUSION}

In conclusion, we have demonstrated that $\mathrm{x}$-ray beam oscillations originating from power line harmonic disturbance of the electron beam in the storage ring can be used as a perturbation signal for ESF control of the HFM steering. Feedback performance is characterized by convergence to within $7 \%$ away from peak position alignment and $1.5 \%$ of peak intensity with characteristic convergence times as low as $33 \mathrm{~ms}$. The results presented here are not beam line specific and can be applied to any beam line where phase stable position oscillations originating in the electron beam are present.

\section{ACKNOWLEDGMENTS}

Work at the Argonne National Laboratory was supported through interagency agreement with the U.S. National Institutes of Health under U.S. Department of Energy Contract No. DEAC02-06CH11357.

\section{APPENDIX: PROCEDURE FOR ESTIMATING OSCILLATION AMPLITUDE}

To estimate the horizontal oscillation amplitude $\psi^{\prime}$, the beam intensity and lock-in signal are recorded as the HFM steering scans the beam across the $5 \mu \mathrm{m}$ aperture (Fig. 5). In the presence of horizontal oscillations, the voltage from the intensity detector downstream from the sample is

$$
V=\left(V_{0}+b \sin \omega t\right) e^{\left[-\frac{1}{2}\left(\frac{\psi_{H}+\psi^{\prime} \sin \left(\omega_{r} t\right)-\psi_{0}}{\sigma}\right)^{2}\right]}
$$

where $V_{0}$ is the sensor voltage at optimal alignment and $b$ is a phenomenological term that accounts for the effects $1.8 \mathrm{kHz}$ intensity oscillations that are independent of the HFM steering angle. Such oscillations could occur due to clipping of the beam close to the source or due to x-ray wavelength oscillations in the insertion device. The lock-in detects the amplitude of the component oscillating at $\sin (\omega t)$ and mixes it down to dc. Expanding Eq. (1) and keeping terms of order $\sin (\omega \mathrm{t})$ yields 


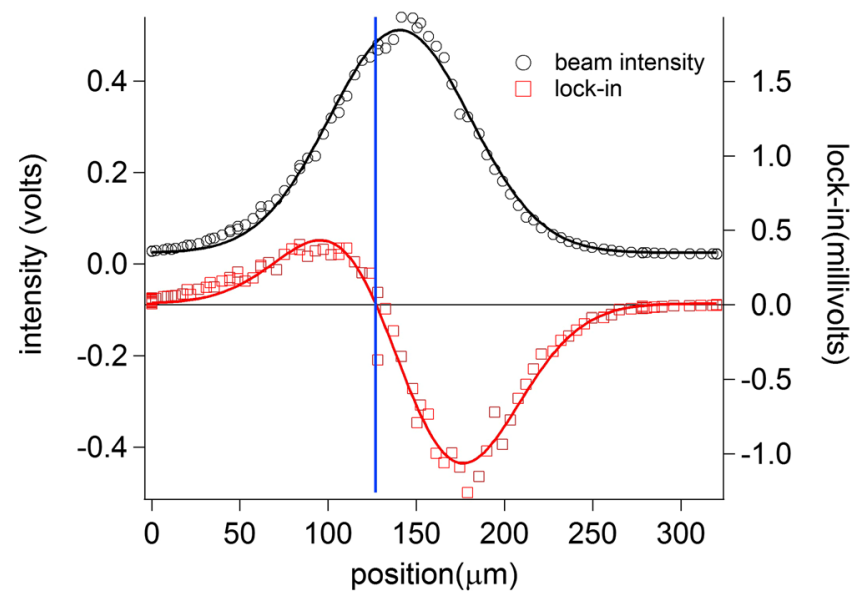

FIG. 5. The beam intensity through the $5 \mu \mathrm{m}$ aperture as the HFM steering is scanned. The intensity FWHM is $93 \mu \mathrm{m}$. The lock-in signal referenced to $360 \mathrm{~Hz}$ is simultaneously recorded. The horizontal oscillation amplitude can be extracted by comparing their amplitude ratio. The vertical line crosses through where the lock-in signal is zero.

$$
V_{L}=\left[\left(\frac{\psi_{H}-\psi_{0}}{\sigma^{2}}\right) \psi^{\prime}+b\right] V
$$

where $V_{L}$ is the lock-in signal. When the oscillation amplitude is small with respect to the beam size, $V_{0}$, $\psi_{0}$, and $\sigma$ can be estimated by fitting the beam intensity in Fig. 5 using Eq. (1) with $\psi^{\prime}$ set to zero. The oscillation amplitude $\psi^{\prime}$ is then estimated by fitting lock-in signal to Eq. (2). Extremum seeking feedback aims to configure the horizontal steering such the $V_{L}$ is zero. When $b$ is zero, Eq. (2) predicts a purely antisymmetric lock-in signal, and the $V_{L}$ zero crossing corresponds to maximum intensity. When $b$ is nonzero and $\psi^{\prime}$ zero, the lock-in traces out the beam profile. When both $b$ and $\psi^{\prime}$ are nonzero the lock-in signal is neither antisymmetric nor symmetric, but rather asymmetric, and the $V_{L}$ zero crossing no longer coincides with maximum intensity. Adding an offset between the lock-in and integrator compensates this effect, vertically shifting the lock-in curve in Fig. 5 until the zero points coincide with the position maximum. The drawback of the offset is that the piezo drive voltage swings instead of pausing when the beam is removed from the detector downstream from the sample.

[1] S. O. Hruszkewycz, T. Fujita, M. W. Chen, and T. C. Hufnagel, Selected area nanodiffraction fluctuation electron microscopy for studying structural order in amorphous solids, Scr. Mater. 58, 303 (2008).

[2] S. Xu, O. Makarov, R. Benn, D. W. Yoder, S. Stepanov, M. Becker, S. Corcoran, M. Hilgart, V. Nagarajan, C. M. Ogata, S. Pothineni, R. Sanishvili, J. L. Smith and R. F. Fischetti, AIP Conf. Proc. 1234, 905 (2010).
[3] G. Evans, L. Alianelli, M. Burt, A. Wagner, and K. J. S. Sawhney, AIP Conf. Proc. 879, 836 (2007)..

[4] X. M. Cheng and D. J. Keavney, Studies of nanomagnetism using synchrotron-based X-ray photoemission electron microscopy (X-PEEM), Rep. Prog. Phys. 75, 026501 (2012).

[5] S. Bonetti, R. Kukreja, Z. Chen, D. Spoddig, K. Ollefs, C. Schöppner, R. Meckenstock, A. Ney, J. Pinto, R. Houanche, J. Frisch, J. Stöhr, H. A. Dürr, and H. Ohldag, Microwave soft X-ray microscopy for nanoscale magnetization dynamics in the 5-10 GHz frequency range, Rev. Sci. Instrum. 86, 093703 (2015).

[6] G. E. Sterbinsky, P. J. Ryan, J.-W. Kim, E. Karapetrova, J. X. Ma, J. Shi, and J.C. Woicik, Local atomic and electronic structures of epitaxial strained $\mathrm{LaCoO}_{3}$ thin films, Phys. Rev. B 85, 020403(R) (2012).

[7] R. A. Rosenberg, S. P. Frigo, and J. K. Simons, Synchrotron radiation induced reactions on surfaces: mechanisms and applications, Appl. Surf. Sci. 79-80, 47 (1994).

[8] N. Shirato, M. Cummings, H. Kersell, Y. Li, B. Stripe, D. Rosenmann, S.-W. Hla, and V. Rose, Elemental fingerprinting of materials with sensitivity at the atomic limit, Nano Lett. 14, 6499 (2014).

[9] S. Matsuyama, H. Nakamori, T. Goto, T. Kimura, K. P. Khakurel, Y. Kohmura, Y. Sano, M. Yabashi, T. Ishikawa, Y. Nishino, and K. Yamauchi, Nearly diffraction-limited $\mathrm{X}$-ray focusing with variable-numerical-aperture focusing optical system based on four deformable mirrors, Sci. Rep. 6, 24801 (2016).

[10] R. P. Winarski, M. V. Holt, V. Rose, P. Fuesz, D. Carbaugh, C. Benson, D. Shu, D. Kline, G. B. Stephenson, I. McNulty, and J. Maser, A hard X-ray nanoprobe beamline for nanoscale microscopy, J. Synchrotron Radiat. 19, 1056 (2012).

[11] D. W. Yoder, O. Makarov, S. Corcoran, and R. F. Fischetti, PID feedback control of monochromator thermal stabilization, Nucl. Instrum. Methods Phys. Res., Sect. A 649, 107 (2011)

[12] D. Mills and V. Pollock, Stabilizing feedback system for synchrotron radiation monochromators, Rev. Sci. Instrum. 51, 1664 (1980).

[13] R. Fischetti, S. Stepanov, G. Rosenbaum, R. Barrea, E. Black, D. Gore, R. Heurich, E. Kondrashkina, A. J. Kropf, S. Wang, K. Zhang, T. C. Irving, and G. B. Bunker, The BioCAT undulator beamline 18ID: a facility for biological non-crystalline diffraction and X-ray absorption spectroscopy at the Advanced Photon Source, J. Synchrotron Radiat. 11, 399 (2004).

[14] S. Stoupin, F. Lenkszus, R. Laird, K. Goetze, K.-J. Kim, and Y. Shvyd'ko, Nanoradian angular stabilization of X-ray optical components, Rev. Sci. Instrum. 81, 055108 (2010).

[15] L. J. van Mallaert and G. H. Schwuttke, FeedbackControlled X-ray diffraction topography, Phys. Status Solidi A 3, 687 (1970).

[16] R. van Silfhout, A. Kachatkou, E. Groppo, C. Lamberti, and W. Bras, Position and flux stabilization of X-ray beams produced by double-crystal monochromators for EXAFS scans at the titanium K-edge, J. Synchrotron Radiat. 21, 401 (2014). 
[17] J. A. Golovchenko, R. A. Levesque, and P. L. Cowan, $\mathrm{X}$-ray monochromator system for use with synchrotron radiation sources, Rev. Sci. Instrum. 52, 509 (1981).

[18] A. Krolzig, G. Materlik, M. Swars, and J. Zegenhagen, A feedback control system for synchrotron radiation double crystal instruments, Nucl. Instrum. Methods Phys. Res., Sect. A 219, 430 (1984).

[19] S. Kim, G. Decker, C. Doose, R. Hogrefe, D. Mangra, R. Merl, and S. Milton, Investigation of low-frequency beam motion at the Advanced Photon Source, Rev. Sci. Instrum. 67, 3371 (1996).

[20] E. Schuster, N. Torres, and C. Xu, Extremum seeking adaptive control of beam envelope in particle accelerators, in IEEE Conference on Control Applications, Munich, Germany, 2006, pp. 1837-1842.

[21] E. Schuster, C. Xu, N. Torres, E. Morinaga, C. Allen, and M. Krstic, Beam matching adaptive control via extremum seeking, Nucl. Instrum. Methods Phys. Res., Sect. A 581, 799 (2007).

[22] M. Krstic and H.-H. Wang, Stability of extremum seeking feedback for general nonlinear dynamic systems, Automatica 36, 595 (2000).

[23] S. Zohar, N. Venugopalan, D. Kissick, M. Becker, S. Xu, O. Makarov, S. Stepanov, C. Ogata, R. Sanishvili, and R. F. Fischetti, Rapid in situ X-ray position stabilization via extremum seeking feedback, J. Synchrotron Radiat. 23, 443 (2016).

[24] S.-J. Liu and M. Krstic, Stochastic Averaging and Stochastic Extremum Seeking (Springer New York, 2012).

[25] S.-J. Liu and M. Krstic, Stochastic averaging in continuous time and its applications to extremum seeking, IEEE Trans. Autom. Control 55, 2235 (2010).
[26] H. Li, J. Peng, W. Liu, Z. Huang, and K.-C. Lin, A Newton-based extremum seeking MPPT method for photovoltaic systems with stochastic perturbations, Int. J. Photoenergy 2014, 1 (2014).

[27] F. Castanos and C. Kunusch, Ditherless extremum seeking for Hydrogen minimization in PEM fuel cells, IEEE Transactions on Industrial Electronics 62, 5218 (2015).

[28] R. F. Fischetti, D. W. Yoder, S. Xu, S. Stepanov, O. Makarov, R. Benn, S. Corcoran, W. Diete, M. SchwoererBoehing, R. Signorato, and others, Optical performance of the GM/CA-CAT canted undulator beamlines for protein crystallography, AIP Conf. Proc. 879, 754 (2007).

[29] R. F. Fischetti, S. Xu, D. W. Yoder, M. Becker, V. Nagarajan, R. Sanishvili, M. C. Hilgart, S. Stepanov, O. Makarov, and J. L. Smith, Mini-beam collimator enables microcrystallography experiments on standard beamlines, J. Synchrotron Radiat. 16, 217 (2009).

[30] S. Xu, L. J. Keefe, A. Mulichak, L. Yan, E. E. Alp, J. Zhao, and R. F. Fischetti, Mini-beam collimator applications at the Advanced Photon Source, Nucl. Instrum. Methods Phys. Res., Sect. A 649, 104 (2011).

[31] S. Xu, V. Nagarajan, R. Sanishvili, and R. F. Fischetti, Alignment protocol for effective use of hard X-ray Quad collimator for micro-crystallography, Proc. SPIE 8125, 81250X (2011).

[32] Y. Tan, W. H. Moase, C. Manzie, D. Nesic, and I. M. Y. Mareels, Extremum Seeking From 1922 To 2010, in Proceedings of the 29th Chinese Control Conference (IEEE, Beijing, 2010), pp. 14-26. 